\title{
Exposants de Łojasiewicz pour les fonctions semi-algébriques
}

\author{
par Azzeddine FeKAK (Casablanca)
}

\begin{abstract}
We prove the rationality of the Łojasiewicz exponent for semialgebraic functions without compactness hypothesis. In the parametric situation, we show that the parameter space can be divided into a finite number of semialgebraic sets on each of which the Lojasiewicz exponent is constant.
\end{abstract}

1. Introduction. Soit $X$ un ensemble semi-algébrique fermé de $R^{n}$ (où $R$ est un corps réel clos), $f$ et $g$ deux fonctions semi-algébriques sur $X$. Si l'ensemble des zéros de $g$ est contenu dans l'ensemble des zéros de $f$, alors le théorème des zéros réels ([1], Théorème 2.6.6) nous dit qu'il existe un entier $n$ et une fonction semi-algébrique $H$ continue sur $X$ tels qu'on ait $f^{n}=H g$ sur $X$. On dira qu'un nombre rationnel $\theta$ verifie la propriété $(\mathrm{P})$ s'il existe une fonction semi-algébrique $h$ continue sur $X$ telle que $|f(x)|^{\theta} \leq h(x)|g(x)|$ pour tout $x \in X$. Dans ce travail nous nous intéressons à la borne inférieure, notée $l_{X}(f, g)$, des nombres rationnels $\theta$ vérifiant $(\mathrm{P})$. Les résultats que nous obtenons sont:

A. La rationalité de cette borne inférieure, appelée l'exposant de Lojasiewicz, et le fait que cet exposant vérifie la propriété $(\mathrm{P})$.

B. Le fait que dans une situation paramétrée on peut découper l'espace en un nombre fini de morceaux semi-algébriques pour lesquels l'exposant de Lojasiewicz est constant, et vérifie la propriété $(\mathrm{P})$. La fonction $h$ de l'inégalité est continue simultanément par rapport aux variables et aux paramètres.

C. Le fait que l'on peut trouver une courbe semi-algébrique sur laquelle l'exposant de Łojasiewicz coïncide avec celui obtenu sur l'ensemble semialgébrique de départ.

1991 Mathematics Subject Classification: Primary 14G30.

Key words and phrases: semialgebraic, Łojasiewicz inequality, real spectrum, Nullstellensatz. 
Ces résultats étendent et complètent, dans le cas réel global (semialgébrique), des travaux classiques sur l'inégalité et les exposants de Łojasiewicz (cf. [2]-[5] et [7]) dans le cas complexe ou analytique local.

On utilise des techniques et des résultats de la géométrie semi-algébrique sur un corps réel clos, et la compacité du spectre réel ([1]).

2. La rationalité de l'exposant de Łojasiewicz. Soit $X$ un ensemble semi-algébrique fermé de $R^{n}$, et $f$ une fonction semi-algébrique. On note $z(f)=\{x \in X \mid f(x)=0\}$. On désigne par $C^{0}(X)$ l'ensemble des fonctions semi-algébriques continues sur $X$.

RÉsultat A. Soient $f$ et $g$ deux fonctions semi-algébriques telles que $z(g) \subset z(f)$. On pose

$$
l_{X}(f, g)=\inf \left\{\left.\theta \in \mathbb{Q}^{+}\left|\exists h \in C^{0}(X) \forall x \in X:\right| f(x)\right|^{\theta} \leq h(x)|g(x)|\right\} .
$$

Alors :

(i) $l_{X}(f, g)=p / q$ est un nombre rationnel.

(ii) $\exists h \in C^{0}(X) \forall x \in X:|f(x)|^{p / q} \leq h(x)|g(x)|$.

Nous donnons la preuve de ce résultat à la fin de ce paragraphe. Nous énonçons tout d'abord deux corollaires.

Rappelons que si $X$ est un ensemble semi-algébrique, la fonction $t \rightarrow$ $d(t, X)$ (où $d(t, X)$ désigne la distance euclidienne de $t$ à $X$ ) est une fonction semi-algébrique. On a les corollaires suivants :

COROLlaire 2.1. Soit $g$ une fonction semi-algébrique sur un ensemble semi-algébrique fermé $X$. On pose $Z=z(g)$, et soit

$$
l_{X}(g)=\inf \left\{\theta \in \mathbb{Q}^{+}\left|\exists h \in C^{0}(X) \forall x \in X: d(x, Z)^{\theta} \leq h(x)\right| g(x) \mid\right\} .
$$

Alors :

(i) $l_{X}(g)=p / q$ est un nombre rationnel.

(ii) $\exists h \in C^{0}(X) \forall x \in X: d(x, Z)^{p / q} \leq h(x)|g(x)|$.

Corollaire 2.2. Soient $A$ et $B$ deux sous-ensembles semi-algébriques fermés dans $X$. On pose

$l(A, B)=\inf \left\{\theta \in \mathbb{Q}^{+} \mid \exists h \in C^{0}(A) \forall x \in A: d(x, A \cap B)^{\theta} \leq h(x) d(x, B)\right\}$.

Alors :

(i) $l(A, B)=p / q$ est un nombre rationnel.

(ii) $\exists h \in C^{0}(A) \forall x \in A: d(x, A \cap B)^{p / q} \leq h(x) d(x, B)$.

Proposition 2.3. Soit $f$ une fonction semi-algébrique sur un intervalle 10,1] non identiquement nulle sur un voisinage de 0 . Il existe un nombre rationnel $p / q$ et une fonction $\varphi$ semi-algébrique continue sur un voisinage $\left[0, \varepsilon\left[\right.\right.$ avec $\varphi(0) \neq 0$ tels que $f(x)=x^{p / q} \varphi(x)$ pour $x$ suffisamment petit. 
Preuve. Dans le cas où $R=\mathbb{R}$ : La fonction $f$ est semi-algébrique sur $] 0,1]$. Il existe un polynôme $P(x, y)$ non identiquement nul tel que $P(x, f(x))=0$. La fonction $f$ est développable au voisinage de zéro en une série de Puiseux réelle [8]. On peut écrire $f(x)=c x^{p / q}(1+\psi(x))$ pour $x$ suffisamment petit avec $\psi(x)$ une série de Puiseux à exposants tous strictement positifs.

En posant $\varphi(x)=c(1+\psi(x))$, on a bien la proposition.

Dans le cas où $R$ est un corps réel clos quelconque:

Rappels : Soit $A$ un anneau commutatif unitaire. Un cône $\alpha$ de $A$ est une partie de $A$ contenant les sommes de carrés d'éléments de $A$ et stable par addition et multiplication. On dit que $\alpha$ est premier si pour tout $a$ et $b$ appartenent à $A$ on a l'implication $a b \in \alpha$ et $a \in \alpha \Rightarrow-b \in \alpha$. On appelle support de $\alpha$ l'idéal premier $l_{\alpha}=\alpha \cap-\alpha$, et on note $k(\alpha)$ la clôture réelle de $k(I)$ pour l'ordre induit par $\alpha$ (voir [1]).

Dans $R(X)$, le corps des fractions rationnelles à une indéterminée, on choisit l'ordre qui rend $X$ positif et plus petit que tout nombre réel strictement positif. Cet ordre est noté $0^{+}$. Le corps $k\left(0^{+}\right)$, la clôture réelle de $R(x)$ pour cet ordre, est le corps des séries de Puiseux algébriques en $X$ à coefficients dans $R$ (voir [1], page 123).

On peut évaluer la fonction $f$ en $0^{+}$(cf. [1], Chap. 7) : $f\left(0^{+}\right) \in k\left(0^{+}\right)$ est une série de Puiseux algébrique en $X$.

On peut écrire $f\left(0^{+}\right)=a X^{p / q}(1+\psi(X))$ avec $\psi(X)$ une série de Puiseux algébrique à exposants tous strictement positifs qui est, pour l'ordre sur $k\left(0^{+}\right)$, plus petite que tout nombre réel positif. D'après la proposition 7.4.1 de [1] il existe pour tout $\delta \in R, \delta>0$, un intervalle ]0, $\varepsilon$ [ tel que pour tout $x \in] 0, \varepsilon\left[\right.$ on ait $a(1-\delta) x^{p / q} \leq f(x) \leq a(1+\delta) x^{p / q}$.

On pose $\varphi(x)=f(x) / a x^{p / q}$ si $x \neq 0$ et $\varphi(0)=a \neq 0$. La fonction $\varphi$ est continue. On a donc la proposition.

Notation. Soient $f$ et $g$ deux fonctions semi-algébriques sur ]0,1]. On note $f \sim g$ si $f(x)$ et $g(x)$ sont équivalentes sur un voisinage de 0 .

COROllaire 2.4. Soit $f$ une fonction semi-algébrique sur $] 0,1]$ non identiquement nulle sur un voisinage de 0 . On pose

$$
\left.\left.\eta=\inf \left\{\theta \in \mathbb{Q}^{+} \mid \exists c>0 \exists \varepsilon>0 \forall x \in\right] 0, \varepsilon\right]:|f(x)| \geq c x^{\theta}\right\} .
$$

Alors :

(i) $\eta=p / q$ est un nombre rationnel.

(ii) Il existe $c>0$ tel que $f(x) \sim c x^{p / q}$.

Preuve. D'après la proposition précédente, il existe un nombre rationnel $p / q \in \mathbb{Q}$ et une fonction $\varphi$ semi-algébrique continue au voisinage de 0 , tels que $f(x)=x^{p / q} \varphi(x)$ pour $x$ suffisamment petit et $\varphi(0) \neq 0$. 
On va montrer que $\eta=p / q$. Si $|f(x)| \geq c x^{\theta}$, on a $x^{p / q} \varphi(x) \geq c x^{\theta}$, d'où $p / q \leq \theta$ puisqe $\varphi(0) \neq 0$.

Dans l'autre sens : $\operatorname{Si} \varphi(0)=a$ on a $|f(x)| \geq(a / 2) x^{p / q}$ pour $x$ suffisamment petit.

Proposition 2.5. Soit $f$ et $g$ deux fonctions semi-algébriques sur un ensemble semi-algébrique fermé $X$ avec $z(g) \subset z(f)$. On pose

$\eta=\inf \left\{\theta \in \mathbb{Q}^{+} \mid f^{\theta} / g\right.$ prolongée par 0 quand $f(x)=0$ est continue sur $X\}$.

Alors $\eta$ est un nombre rationnel.

Pre u ve. Posons, pour $x \in X$ et $u \in R, K_{x, u}=\{y \in X \mid\|x-y\| \leq 1$ et $|f(y)|=u\}$, et définissons la fonction $v(x, u)$ par

$$
v(x, u)= \begin{cases}\inf \left\{|g(y)| \mid y \in K_{x, u}\right\} & \text { si } K_{x, u} \neq \emptyset, \\ 1 & \text { si } K_{x, u}=\emptyset .\end{cases}
$$

La fonction $v$ est semi-algébrique sur $X \times R$. On remarque que pour tout $x$, si $u \neq 0$, alors $v(x, u) \neq 0$. Pour tout nombre rationnel $p / q$, on pose

$$
C_{p / q}=\left\{x_{0} \in z(f) \mid \exists c\left(x_{0}\right)>0: v\left(x_{0}, u\right) \sim c u^{p / q}\right\} .
$$

L'ensemble $C_{p / q}$ est un ensemble semi-algébrique de $R^{n}$; on note $\widetilde{C}_{p / q}$ le constructible qui lui est associé par l'identification tilda (cf. [1], Chap. 7).

Soit $\alpha \in \operatorname{Spec}_{r}\left(R\left[X_{1}, \ldots, X_{n}\right]\right)$ un cône premier. La fonction $v$ peut s'étendre en une fonction semi-algébrique $v_{k(\alpha)}: X_{k(\alpha)} \times k(\alpha) \rightarrow k(\alpha)$ où $X_{k(\alpha)}$ est l'extension de $X$ à $k(\alpha)$.

En prenant $\alpha \in \widetilde{z(f)}$ et en appliquant le corollaire 2.4 sur le corps réel clos $k(\alpha)$, il existe $c(\alpha) \geq 0$ et $p / q \in \mathbb{Q}$ tel que

$$
v_{k(\alpha)}(x(\alpha), u) \sim c(\alpha) u^{p / q} .
$$

On a donc $\alpha \in \widetilde{C}_{p / q}$. Ceci montre que les $\widetilde{C}_{p / q}$ forment un recouvrement de $\widetilde{z(f)}$. D'après la compacité de la topologie du spectre réel [1], $z(f)$ est recouvert par un nombre fini de $C_{p / q}$. D'où le nombre fini des $p / q$. En prenant le plus petit $p / q$ quand $x_{0}$ varie dans $z(f)$, la formule suivante est vérifiée :

$$
\begin{array}{ll}
\forall x_{0} \in z(f) \exists c\left(x_{0}\right)>0: & \left|v\left(x_{0}, u\right)\right| \geq c\left(x_{0}\right) u^{p / q}, \\
\exists a_{0} \in z(f) \exists c\left(a_{0}\right)>0: & v\left(a_{0}, u\right) \sim c\left(a_{0}\right) u^{p / q} .
\end{array}
$$

On va montrer que $\eta=p / q$.

La formule $(*)$ entraîne que sur $L=\left\{y \in X \mid\left\|y-x_{0}\right\| \leq 1\right.$ et $\left.f(y) \neq 0\right\}$ on a

$(* *) \quad c\left(x_{0}\right)|f(y)|^{p / q} \leq|g(y)|$ pour $|f(y)|$ assez petit. 
Pour tout nombre rationnel $\varepsilon>0$ la fonction $f^{\varepsilon+p / q} / g$ prolongée par 0 quand $f(x)=0$ est continue sur $X$, d'où $\eta \leq p / q$.

Si $f^{\theta} / g$ prolongée par 0 quand $f(x)=0$ est continue sur $X$, alors $f^{\theta} / g$ est bornée au voisinage de $a_{0}$ de la formule $(*)$, donc sur ce voisinage on a $|g| \geq c|f|^{\theta}$. Ceci entraîne que $p / q \leq \theta$, d'où $p / q \leq \eta$, et par suite $p / q=\eta$.

Preuve du résultat A. (i) On pose $p / q=\inf \left\{\theta \in \mathbb{Q}^{+} \mid f^{\theta} / g\right.$ prolongée par 0 quand $f(x)=0$ est continue sur $X\}$. On va montrer que $l_{X}(f, g)=p / q$.

Si $|f|^{\theta} \leq h|g|$ sur $X$, alors pour tout nombre rationnel $\varepsilon$ positif $f^{\theta+\varepsilon} / g$ prolongée par 0 quand $f(x)=0$ est continue sur $X$, donc $p / q \leq \theta+\varepsilon$ pour tout $\varepsilon$, et par suite $p / q \leq l_{X}(f, g)$.

Pour montrer l'autre inégalité il suffit de montrer (ii) pour l'exposant $p / q$. On pose

$$
k(x)= \begin{cases}f(x)^{p / q} / g(x) & \text { si } f(x) \neq 0 \\ 0 & \text { si } f(x)=0\end{cases}
$$

Comme la fonction $k$ est semi-algébrique localement bornée sur $X$ (d'après la formule $(* *)$ de la preuve de la proposition 2.5 ), on peut la majorer par un polynôme $h$ sur $X$ tout entier. On a donc pour tout $x \in X$

$$
|f(x)|^{p / q} \leq h(x)|g(x)| \text {. }
$$

3. Inégalités de Łojasiewicz avec paramètre. Nous nous intéressons dans ce paragraphe aux exposants de Łojasiewicz pour des familles de fonctions semi-algébriques continues dépendants d'un paramètre.

Si $X$ est un ensemble semi-algébrique de $R^{n} \times R^{p}$, on peut considérer $X$ comme une famille de sous-ensembles de $R^{n}$ paramétrisée dans $R^{p}$. La fibre de $X$ au point $t$ de $R^{p}$ est $X_{t}=\left\{x \in R^{n} \mid(x, t) \in X\right\}$.

La restriction de la famille $X$ au sous-ensemble $S$ de $R^{p}$ est $X \mid S=$ $X \cap\left(R^{n} \times S\right)$.

Si $f: X \rightarrow R$ est une fonction semi-algébrique sur $X \subset R^{n} \times R^{p}$, la fibre de $f$ en $t$ est la fonction semi-algébrique $f_{t}: X_{t} \rightarrow R$ définie par

$$
f_{t}(x)=y \Leftrightarrow f(x, t)=y \text {. }
$$

Soit $\alpha \in R^{p}=\operatorname{Spec}_{r}\left(R\left[X_{1}, \ldots, X_{p}\right]\right)$. On peut aussi définir la fibre de $X$ en $\alpha$ : si $X$ est défini par une formule $\Phi(x, t)$ de premier ordre, alors

$$
X_{\alpha}=\left\{x \in k(\alpha)^{n} \mid \Phi(x, t(\alpha))\right\} \subset k(\alpha)^{n} .
$$

Elle ne dépend que de $X$ et non pas du choix de $\Phi$. La fibre $X_{\alpha}$ est appelée la fibre de la famille $X$ en $\alpha$ ([1], page 125).

Si $f: X \rightarrow R$ est une fonction semi-algébrique, on définit la fibre $f_{\alpha}$ de $f$ au point $\alpha$ de $R^{p}$ par : [graphe $\left.(f)\right]_{\alpha} \subset k(\alpha)^{n} \times k(\alpha)$ est le graphe de $f_{\alpha}: X_{\alpha} \rightarrow k(\alpha)$. Le résultat essentiel dans l'étude des fibres des familles 
d'ensembles et de fonctions semi-algébriques est le fait qu'une propriété exprimable dans le langage du premier ordre des corps ordonnés d'une fibre en $\alpha \in \widetilde{R}^{p}$ reste valable sur un sous-ensemble semi-algébrique $S \subset R^{p}$ tel que $\alpha \in \widetilde{S}$ (cf. [1], proposition 7.4.4).

RÉSUltat B. Soient A un ensemble semi-algébrique fermé de $R^{n} \times R^{p}$, $f(x, t)$ et $g(x, t)$ deux fonctions semi-algébriques continues en $x$ sur $A$ telles que $z(g) \subset z(f)$. Alors il existe une partition finie en ensembles semialgébriques $R^{p}=\bigcup S_{i}$, des fonctions semi-algébriques continues $h_{i}: A \mid S_{i} \rightarrow$ $R$, et des nombres rationnels $p_{i} / q_{i}$ tels que:

(i) $\left.\mid f(x, t))\left.\right|^{p_{i} / q_{i}} \leq h_{i}(x, t) \mid g(x, t)\right)|\operatorname{sur} A| S_{i}$ pour $t \in S_{i}$.

(ii) $p_{i} / q_{i}$ est l'exposant de Eojasiewicz de $f_{t}$ par rapport à $g_{t}$ pour $t \in S_{i}$.

Preuve. On note $f_{t}(x)=f(x, t)$ et $g_{t}(x)=g(x, t)$ les fibres en $t$ de $f$ et $g$. Les fonctions $f_{t}$ et $g_{t}$ sont semi-algébriques sur $A_{t}$. On note $p / q=\eta_{t}$ l'exposant de Łojasiewicz de $f_{t}$ par rapport à $g_{t}$ sur $A_{t}$. Ce $p / q$ vérifie la formule suivante (voir la formule $(*)$ de la preuve de la proposition 2.5) :

$$
\begin{array}{ll}
\forall x_{0} \in z\left(f_{t}\right) \exists c\left(x_{0}\right)>0: & \left|v_{t}\left(x_{0}, u\right)\right| \geq c u^{p / q}, \\
\exists a_{0} \in z\left(f_{t}\right) \exists c\left(a_{0}\right)>0: & v_{t}\left(a_{0}, u\right) \sim c u^{p / q}
\end{array}
$$

où $v_{t}$ est la fonction $v$ obtenue en remplaçant $f$ par $f_{t}$ et $g$ par $g_{t}$.

On va montrer qu'il n'y a qu'un nombre fini d'exposants rationnels $p / q$ qui vérifient cette formule quand $t$ varie dans $R^{p}$.

On raisonne comme dans la preuve de la proposition 2.5. On pose, pour un nombre rationnel $p / q$,

$$
\begin{aligned}
D_{p / q}=\left\{t \in R^{p} \mid \forall x_{0}\right. & \in z\left(f_{t}\right) \exists c>0:\left|v_{t}\left(x_{0}, u\right)\right| \geq c\left(x_{0}\right) u^{p / q}, \\
& \left.\exists a_{0} \in z\left(f_{t}\right) \exists c\left(a_{0}\right)>0: v_{t}\left(a_{0}, u\right) \sim c\left(a_{0}\right) u^{p / q}\right\} .
\end{aligned}
$$

L'ensemble $D_{p / q}$ est un ensemble semi-algébrique de $R^{p}$.

Soit $\alpha \in \widetilde{R}^{p}$. D'aprés la formule (*) de la proposition 2.5 appliquée à $f_{\alpha}$ et $g_{\alpha}$ dans le corps réel clos $k(\alpha)$, il existe un nombre rationnel $p / q$ tel que $\alpha \in \widetilde{D}_{p / q}$. Ceci montre que les $\widetilde{D}_{p / q}$ recouvrent $\widetilde{R}^{p}$ et donc par la compacité de la topologie du spectre réel, l'ensemble des paramètres $R^{p}$ est réunion d'un nombre fini de $D_{p / q}$. De plus, si $\alpha \in \widetilde{D}_{p / q}$, alors l'exposant de Lojasiewicz de $f_{\alpha}$ par rapport à $g_{\alpha}$ est $p / q$ et donc il existe une fonction semi-algébrique continue $H: X_{\alpha} \rightarrow k(\alpha)$ telle que $\left|f_{\alpha}(x)\right|^{p / q} \leq H(x)\left|g_{\alpha}(x)\right|$ $\operatorname{sur} X_{\alpha}$.

La fonction $H$ est la fibre en $\alpha$ d'une fonction semi-algébrique continue $h: X \mid S^{\alpha} \rightarrow R$ ([1], prop. 7.4.8) avec $\alpha \in \widetilde{S}^{\alpha}$, et on peut supposer que $S^{\alpha} \subset$ $D_{p / q}$ et que sur $S^{\alpha}$ on a $|f(x, t)|^{p / q} \leq h(x, t)|g(x, t)|$. Les $\widetilde{S}^{\alpha}$ recouvrent $\widetilde{R}^{p}$, et donc par compacité on peut en extraire un recouvrement fini : $R^{p}=$ $\bigcup_{i \in I} S_{i}$, avec $I$ fini. 
Corollaire 3.1. Soit $g(x, t)$ une fonction semi-algébrique continue en $x$ sur un ensemble semi-algébrique $A \subset R^{p}$. On pose $Z=z(g)$. Alors il existe une partition finie en ensembles semi-algébriques $R^{p}=\bigcup S_{i}$, des fonctions semi-algébriques continues $h_{i}: A \mid S_{i} \rightarrow R$, et des $p_{i} / q_{i}$ tels que:

(i) $d\left(x, Z_{t}\right)^{p_{i} / q_{i}} \leq h_{i}(x, t)\left|g_{t}(x)\right|, \forall x \in A_{t}$ pour $t \in S_{i}$.

(ii) $p_{i} / q_{i}$ est l'exposant de Eojasiewicz de $g_{t}$ par rapport à $d\left(x, Z_{t}\right)$ pour $t \in S_{i}$

Corollaire 3.2. Soient $A$ et $B$ deux ensembles semi-algébriques fermés de $R^{n} \times R^{p}$. Alors il existe une partition finie en ensembles semi-algébriques $R^{p}=\bigcup S_{i}$, des fonctions $h_{i}: A \mid S_{i} \rightarrow R$, et des $p_{i} / q_{i}$ tels que :

(i) $d\left(x, A_{t} \cap B_{t}\right)^{p_{i} / q_{i}} \leq h_{i}(x, t) d\left(x, B_{t}\right), \forall x \in A_{t}$ pour $t \in S_{i}$.

(ii) $p_{i} / q_{i}$ est l'exposant de Eojasiewicz de $d\left(x, B_{t}\right)$ par rapport à $d\left(x, A_{t} \cap B_{t}\right)$ pour $t \in S_{i}$.

4. Exposants de Łojasiewicz et courbes semi-algébriques. Dans ce paragraphe on montrera, dans le cas où $X$ est un ensemble semi-algébrique fermé non nécessairement borné, que l'exposant de Łojasiewicz est atteint sur une courbe semi-algébrique sur $X$. Pour cela on va se ramener tout d'abord au cas fermé borné, puis on passera au cas général en utilisant la démonstration du résultat $\mathrm{A}$. On remarquera que lorsque $X$ est fermé borné on peut remplacer la fonction $h$ de l'inégalité de Łojasiewicz par une constante $c$ appartenant à $R$.

Proposition 4.1 (voir [2], Théorème 1). Soit $K$ un ensemble semialgébrique fermé borné de $R^{n}$, $f$ et $g$ deux fonctions semi-algébriques sur $K$ tel que $\emptyset \neq z(g) \subset z(f)$. On pose

$$
p / q=\inf \left\{\left.\theta \in \mathbb{Q}^{+}|\exists c \in R \forall x \in K:| f(x)\right|^{\theta} \leq c|g(x)|\right\} .
$$

Alors il existe une courbe semi-algébrique $\tau:[0,1[\rightarrow K$ tel que $p / q$ est égal à l'exposant de Eojasiewicz de $f$ par rapport à $g$ calculé sur l'image $\tau([0,1[)$.

Preuve. On peut supposer $f \geq 0$ et $g \geq 0$ sur $K$. Posons

$$
K^{*}=\{u \in K \backslash z(f) \mid f(x)=f(u) \Rightarrow g(x) \geq g(u)\} .
$$

L'ensemble $K^{*}$ est semi-algébrique borné.

Soit $a \in f^{-1}(0)$ tel que $a$ soit adhérent à $K^{*}$ (un tel $a$ existe puisque $K$ est fermé borné). D'après le lemme de sélection de courbes ([1], théorème 2.2.5) il existe une courbe semi-algébrique $\tau:[0,1] \rightarrow K$ telle que $\tau(0)=a$ et $\tau(t) \in K^{*}$ pour $\left.t \in\right] 0,1$. On peut écrire, d'après la proposition 2.3, $f \circ \tau(t)=t^{\alpha} \varphi_{1}(t)$ et $g \circ \tau(t)=t^{\beta} \varphi_{2}(t)$ avec $\varphi_{1}(0), \varphi_{2}(0)$ non nuls et $\alpha, \beta$ deux nombres rationnels positifs. 
Il suffit de montrer que $p / q=\beta / \alpha$ pour prouver la proposition. On a évidemment $p / q \geq \beta / \alpha$, car $\beta / \alpha$ est l'exposant de Łojasiewicz pour la restriction de $f$ et $g$ à $\tau([0, \varepsilon[)$ pour $\varepsilon$ convenable.

Choisissons $r>0$ tel que $f^{-1}(\zeta) \cap \tau([0, \varepsilon[) \neq \emptyset$ pour $\zeta \in[0, r]$. Comme $K$ est fermé borné il suffit de se borner aux $x \in K$ tels que $f(x) \in[0, r]$ (rappelons que par hypothèse $z(g) \subset z(f)$ ); mais pour un tel $x$, il existe $u \in \tau([0, \varepsilon[)$ tel que $f(x)=f(u)$ et $g(x) \geq g(u)$, d'où l'assertion puisqu'on a choisi $\varepsilon$ assez petit pour que $\beta / \alpha$ soit l'exposant de Lojasiewicz de $f$ par rapport à $g$ sur la courbe $\tau([0, \varepsilon[)$. Quitte à dilater l'intervalle $[0, \varepsilon[$, on peut prendre $\varepsilon=1$.

RÉSUlTAT C. Soit $f$ et $g$ deux fonctions semi-algébriques sur un ensemble semi-algébrique fermé $X$ avec $z(g) \subset z(f)$. Il existe une courbe semialgébrique $\tau:\left[0,1\left[\rightarrow X\right.\right.$ tel que $l_{X}(f, g)$ est égal à l'exposant de Eojasiewicz de la restriction de $f$ et $g$ sur $\tau([0,1[)$.

Preuve. Posons $l_{X}(f, g)=p / q$. Ce nombre rationnel $p / q$ vérifie la formule $(*)$ de la preuve de la proposition 2.5 .

Il existe $a_{0} \in X$ avec $f\left(a_{0}\right)=0$ tel que sur $L=\left\{y \in X \mid\left\|y-x_{0}\right\| \leq 1\right.$ et $f(y) \neq 0\}$ on a $c\left(a_{0}\right)|f(y)|^{p / q} \leq|g(y)|$ pour $|f(y)|$ assez petit. Il existe un $\varepsilon>0$ tel que $p / q$ est l'exposant de Łojasiewicz de la restriction de $f$ et $g$ sur l'ensemble $L_{\varepsilon}=\left\{y \in X \mid\left\|y-a_{0}\right\| \leq \varepsilon\right.$ et $\left.f(y) \neq 0\right\}$ (en choisissant $\varepsilon$ assez petit $|f|^{p / q} /|g|$ est majorée sur $L_{\varepsilon}$ ). C'est aussi l'exposant de Łojasiewicz de la restriction de $f$ et $g$ sur l'ensemble fermé borné $K=\left\{y \in X \mid\left\|y-a_{0}\right\| \leq \varepsilon\right\}$.

D'après la proposition précédente, il existe une courbe semi-algébrique tracée sur $K$, donc sur $X$, sur laquelle $l_{X}(f, g)=p / q$ est l'exposant de Łojasiewicz de la restriction de $f$ et $g$ à $\tau([0, l[)$.

Corollaire 4.2. Soit $f(x, t)$ et $g(x, t)$ deux fonctions semi-algébriques continues en $x$ sur $X$ de $R^{n} \times R^{p}$ avec $z(g) \subset z(f)$. Alors il existe une partition finie en ensembles semi-algébriques de la projection de $X$ sur $R^{p}$ telle que l'exposant de Lojasiewicz $l_{S_{i}}(f, g)$ sur $X \mid S_{i}$ est égal à l'exposant de Eojasiewicz calculé sur une famille de courbes semi-algébriques $\Gamma:\left[0,1\left[\times S_{i} \rightarrow X \mid S_{i}\left(\Gamma\right.\right.\right.$ commutant avec la projection sur $\left.S_{i}\right)$.

Preuve. On note $l\left(f_{\alpha}, g_{\alpha}\right)$ l'exposant de Lojasiewicz de $f_{\alpha}$ par rapport à $g_{\alpha}$ sur $X_{\alpha}$. D'après la proposition précédente, il existe une courbe semialgébrique $\gamma:] 0,1\left[k_{(\alpha)} \rightarrow X_{\alpha}\right.$ sur laquelle l'exposant est atteint, $\gamma$ est la fibre en $\alpha$ d'une famille de courbes $\Gamma:\left[0,1\left[\times S^{\alpha} \rightarrow X \mid S^{\alpha}\right.\right.$ où $\Gamma$ est semialgébrique continue, avec $\alpha \in \widetilde{S}^{\alpha}$. Quitte à restreindre $S^{\alpha}$, on peut supposer que l'exposant $l\left(f_{t}, g_{t}\right)$ reste constant et égal à $l\left(f_{\alpha}, g_{\alpha}\right)$ pour $t \in S^{\alpha}$, et que cet exposant est atteint sur la courbe $\Gamma_{t}$. 
Les $\widetilde{S}^{\alpha}$ recouvrent le tilda de la projection de $X$ sur $R^{p}$, donc par compacité de la topologie du spectre réel il existe un nombre fini de ces $S^{\alpha}$ qui recouvrent la projection de $X$ sur $R^{p}$.

\section{Références}

[1] J. Bochnak, M. Coste et M. F. Roy, Géométrie algébrique réelle, Ergeb. Math. Grenzgeb. (3) 12, Springer, 1987.

[2] J. Bochnak et J. J. Risler, Sur les exposants de Eojasiewicz, Comment. Math. Helv. 50 (1975), 493-507.

[3] T.-C. Kuo, Computation of Eojasiewicz exponent of $f(x, y)$, ibid. 49 (1974), 201-213.

[4] M. Lejeune et B. Teissier, Dépendance intégrale sur les idéaux et équisingularité, Séminaire Ecole Polytechnique, Publ. Inst. Fourier, 1974.

[5] B. Lichtin, Estimation of Eojasiewicz exponents and Newton polygons, Invent. Math. 64 (1981), 417-429.

[6] S. Łojasiewicz, Ensembles semi-analytiques, notes multigraphiées, I.H.E.S., 1965.

[7] G. Raby, Théorème des zéros sous-analytiques et inégalités de Eojasiewicz, dans : Lecture Notes in Math. 1028, Springer, 1983, 253-265.

[8] R. Walker, Algebraic Curves, Dover, 1962.

ECOLE ROYALE NAVALE

Bd SOUR JDID

CASABLANCA 01, MAROC 